Historic, Archive Document

Do not assume content reflects current scientific knowledge, policies, or practices. 



\section{PRICES FOR SUMMER OF 1911}

All cactus cuttings or plants should be planted in the warm Summer months. Plant one third of the cutting under the soil, two thirds above. After planting keep rather dry until they begin to root or to show new leaf buds after which they will endure more moisture.

Most of these varieties are wholly new and the stock limited, no one on earth having a single plant except these now growing on my experiment farms.

These prices given are for June and July delivery. Twenty-five per cent discount from these prices will be allowed for August and September deliveries. Freight or express charges to be paid by purchaser. All cuttings and plants lightly and carefully packed, no charge for boxes or packing. Smaller cuttings can sometimes be sent by mail.

\section{ROBUSTA and SPECIAL}

Cuttings each $80 \mathrm{c}$; per ten $\$ 4$; per hundred $\$ 30$; per thousand $\$ 200$.

Well rooted plants double these prices.

\section{ELDORADO, COMPETENT, SIGNAL and TITANIA}

Cuttings each $\$ 3$, per ten $\$ 20 \quad$ Rooted plants each $\$ 6$

\section{ACTUAL, ARBITER, BANANA, BUSTER, MARKET, NIAGARA,} ROYAL, QUILLOTA, VERTEX and OPALINE.

Cuttings each $\$ 1.50$; per ten $\$ 10 \quad$ Rocted plants each $\$ 4$

\section{ELEGANT and BIJOU}

Cuttings each $40 \mathrm{c}$; per ten $\$ 2.50$; per hundred $\$ 18$; per thousand $\$ 150$

A Collection of Twenty other very valuable new forage and fruiting varieties for trial. For the twenty new varieties, one each, $\$ 20$

Sixty tons of the common type of so-called "spineless" cactus so often sold as "Burbank's" or "just as good as Burbank's", price

Cuttings each $2 \mathrm{c}$; per ten $10 \mathrm{c}$; per hundred $80 \mathrm{c}$; per thousand $\$ 6$, delivered here. Boxes and packing extra on these old half civilized ones.

\section{WOOLY and BASILARIS}

Cuttings each $60 \mathrm{c}$; per ten $\$ 3$

\section{QUISCO}

Heavily rooted plants two or three pounds each. Six to eight inches high, each $80 \mathrm{c}$; per ten $\$ 4$. Small globular plants one inch in diameter, each $20 \mathrm{c}$; per ten $\$ 1.50$; per hundred $\$ 10$

\section{ECHINOPSIS MULLERI}

Small plants, each $20 \mathrm{c}$; per ten $\$ 1.25$

Large plants, each $60 \mathrm{c}$; per ten $\$ 4.00$

\section{CEREUS PITAJAYA}

Plants, extra fruiting kind, each $\$ 2$

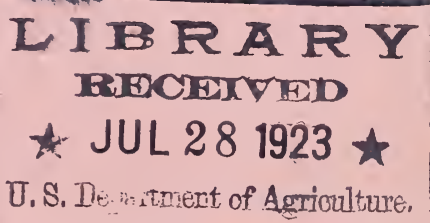

LUTHER BURBANK, Santa Rosa, Sonoma County, California, U. S. A. 
-

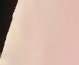

